\title{
ON THE VOLUME OF METRIC BALLS
}

\author{
CHRISTOPHER B. CROKE ${ }^{1}$
}

\begin{abstract}
In this paper we consider metrics of the form $d s^{2}=d r^{2}+f^{2}(r, \theta) d \theta^{2}$ on a ball of dimension $n \geqslant 3$. We show that if the diameters (geodesics through the origin) minimize length then the volume of the ball is larger than the volume of the hemisphere of the corresponding round sphere. This relates to a conjecture first considered by Marcel Berger. We also give examples in all dimensions of radially symmetric metrics on balls of radius $\pi / 2$ having arbitrarily small volume and yet having no pair of points conjugate along a diameter.
\end{abstract}

I. Introduction. Let $M^{n}$ be a complete riemannian manifold of dimension $n$ and injectivity radius $i(M)$. Here we consider the volume of the metric ball, $B(p, r)$, of radius $r$ about a point $p$ of $M$. A fundamental question first asked by $\mathrm{M}$. Berger is

Question A. If $r \leqslant i(M) / 2$, is $\operatorname{Vol}(B(p, r)) \geqslant \frac{1}{2} \alpha(n)(2 r / \pi)^{n}$, where $\alpha(n)$ is the volume of the unit $n$ sphere, with equality characterizing hemispheres?

One can ask the related question about the boundary spheres $S(p, r)=\{q \in M \mid$ $d(p, q)=r\}$.

Question B. If $r \leqslant i(M) / 2$, is $\operatorname{Vol}(S(p, r)) \geqslant \alpha(n-1)(2 r / \pi)^{n-1}$ with equality holding if and only if $B(p, r)$ is isometric to a hemisphere?

A positive answer to Question A implies a positive answer to B by Theorem 11 of [C].

For $M$ compact there are natural questions related to $\mathrm{A}$ and $\mathrm{B}$ for which we know some answers.

The global question is: Is $\operatorname{Vol}(M) \geqslant \alpha(n)(i(M) / \pi)^{n}$ with equality holding if and only if $M$ is isometric to a round sphere?

Question A "on the average" is: Is

$$
\frac{1}{\operatorname{Vol}(M)} \int_{M} \operatorname{Vol}(B(p, r)) d p \geqslant \frac{1}{2} \alpha(n)\left(\frac{2 r}{\pi}\right)^{n},
$$

with equality holding if and only if $M$ is isometric to a round sphere? Question B "on the average" is similar.

The global question was answered positively by Berger in [B3]. The argument in [B3] also answered Question B "on the average" for $r=i(M) / 2$. Previously Berger and Kazdan (see [B-K] or [Be] Appendices D and E) had shown that the global question was true with half the sharp constant (i.e. $\operatorname{Vol}(M)>\frac{1}{2} \alpha(n)(i(M) / \pi)^{n}$ ) by answering Question A "on the average" with half the sharp constant.

Received by the editors October 4, 1982.

1980 Mathematics Subject Classification. Primary 53C65.

${ }^{1}$ Research supported by NSF grant \# MCS79-01780. 
As to Questions A and B themselves, the only known complete answer is for Question B in two dimensions. However, nonsharp answers (i.e., $\operatorname{Vol}(B(p, r)) \geqslant$ $C(n) r^{n}$ for some constant $\left.C(n)\right)$ were shown by Berger for $n=2,3$ in [B1] and [B2], and for all $n$ by the author in [C] (Proposition 14).

The condition that $r \leqslant i(M) / 2$ is a mixture of local and global conditions. One often wants to consider $B(p, r)$ as a riemannian manifold with boundary ignoring the rest of $M$. In this case the appropriate assumption is:

Condition $(\mathrm{G})$. Every geodesic segment in $B(p, r)$ minimizes distance (in $B(p, r)$ ) between the endpoints.

Note that if $r \leqslant i(M) / 2$ then $B(p, r)$ satisfies Condition $\mathrm{G}$ but the converse is not true. The results in [B1, B2, and C] hold for $B(p, r)$ if it satisfies Condition $\mathrm{G}$.

Here we consider only metric balls whose metric in normal polar coordinates $(r, \theta)$ has the special form $d s^{2}=d r^{2}+h^{2}(r, \theta) d \theta^{2}$ where $h(r, \theta)>0$ for $r \neq 0$ and $d \theta^{2}$ represents the standard metric on the unit sphere. By a diameter we will mean a geodesic through $p$ (the center). We show

THEOREM. Let $B(p, R)$ be a metric ball of dimension $n \geqslant 3$ with a metric of the form $d s^{2}=d r^{2}+h^{2}(r, \theta) d \theta^{2}$. Then if all diameters minimize distance between their endpoints we have $\operatorname{Vol}(B(p, R)) \geqslant \frac{1}{2} \alpha(n)(2 R / \pi)^{n}$ with equality characterizing hemispheres.

This says that for metrics of this form and $n \geqslant 3$ the answer to Question A (and hence $\mathrm{B}$ ) is yes.

The proof is an extension of an argument given by Berger in [B4] for the two-dimensional case. The proof however does not work in two dimensions (there is an error in [B4]) but the extension to $n \geqslant 3$ does work. It would be nice to know the theorem in two dimensions for in that case all metrics are of the form $d s^{2}=d r^{2}+$ $h^{2}(r, \theta) d \theta^{2}$; however at the moment this is an open question.

The assumption of the theorem is weaker than Condition $(G)$ since we only assume that diameters minimize. One might well ask if one can further weaken the assumption to: no two interior points on a diameter are conjugate along that diameter. A positive answer to this question, even in the rotationally symmetric case, would, with some further argument, answer Question A in general up to a factor of 2. However here we give

EXAMPLES. For each $\varepsilon>0$ there is a function $g_{\varepsilon}(r)>0, r \in(0, \pi / 2]$, such that $d s^{2}=d r^{2}+g_{\varepsilon}^{2}(r) d \theta^{2}$ is a smooth (rotationally symmetric) metric on a ball $B(p, \pi / 2$ ) such that

(a) $\operatorname{Vol}(B(p, \pi / 2))<\varepsilon$, and

(b) No two (interior) points are conjugate along a diameter.

The same function $g_{\varepsilon}(r)$ works for all dimensions.

II. The proofs.

Proof OF THEOREM. By multiplying the metric by a constant we may assume that $R=\pi / 2$. Let $\left(B_{0}, g_{0}\right)$ represent the unit hemisphere with the standard metric. $B_{0}$ is identified to $B$ (up to diffeomorphism) via the exponential map at the center. 
If $v \in T_{x} B_{0}$ is such that $g_{0}(v, v)=1$ then $g(v, v)=\cos ^{2} \phi+f^{2}(x) \sin ^{2} \phi$, where $\phi$ represents the angle (in $g_{0}$ ) between $v$ and the radial vector, $d / d r$, and $f(x)=$ $f(r, \theta)=h(r, \theta) / \sin (r)$. The volume of $(B, g)$ is given by $\int_{B_{0}} f^{n-1}(x) d_{0} x$, where $d_{0} x$ is the volume form on $\left(B_{0}, g_{0}\right)$.

Let $U_{0}$ be the unit tangent bundle of $\left(B_{0}, g_{0}\right)$ and let $\Gamma$ be the space of geodesics on $B_{0}$ (i.e. $\Gamma$ is the space of oriented half great circles). $U_{0}$ is fibered in two ways. The first fibration is the standard

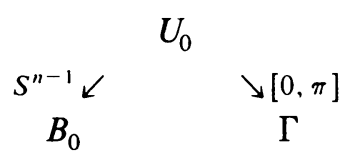

one (the projection to $B_{0}$ takes a unit vector to its base point) where the fiber over $x$ is the unit tangent sphere $U_{x}$. The measure $d u$ on $U_{0}$ is simply the product measure. The second fibration is the one induced by the geodesic flow. Liouville's theorem (see [Be, 1.125]) shows that $\Gamma$ admits a measure $d \gamma$ such that $d u=d t \times d \gamma(d t$ is the standard measure on $[0, \pi])$.

Each $\gamma \in \Gamma$ is a curve from a point on the boundary to the point at the other end of the diameter. Hence by assumption its length in $g$ is $\geqslant \pi$. By a Schwarz inequality we have

$$
\int_{0}^{\pi} g\left(\gamma^{\prime}(t), \gamma^{\prime}(t)\right) d t \geqslant \frac{1}{\pi}\left[\int_{0}^{\pi} \sqrt{g\left(\gamma^{\prime}(t), \gamma^{\prime}(t)\right)} d t\right]^{2} \geqslant \pi
$$

with equality holding if and only if $g\left(\gamma^{\prime}(t), \gamma^{\prime}(t)\right)=1$ for all $t \in[0, \pi]$.

Integrating over $\Gamma$ we get

$$
\begin{aligned}
\frac{1}{2} \alpha(n) \alpha(n-1) & =\pi \operatorname{Vol}(\Gamma) \leqslant \int_{\Gamma} \int_{0}^{\pi} g\left(\gamma^{\prime}(t), \gamma^{\prime}(t)\right) d t d \gamma \\
& =\int_{U_{0}} g(v, v) d u=\int_{B_{0}} \int_{U_{x}} g(v, v) d \theta d_{0} x
\end{aligned}
$$

Where $d \theta$ above represents the standard volume form on $U_{x}$.

For fixed $x \in B_{0}$ we have

$$
\begin{aligned}
\int_{U_{x}} g(v, v) d \theta & =\alpha(n-2) \int_{0}^{\pi}\left(\cos ^{2} \phi+f^{2}(x) \sin ^{2} \phi\right) \sin \phi^{n-2} d \phi \\
& =\alpha(n-1)-\frac{\alpha(n-2) \alpha(n+1)}{\alpha(n)}+f^{2}(x) \frac{\alpha(n-2) \alpha(n+1)}{\alpha(n)} .
\end{aligned}
$$

So integrating over $B_{0}\left(\operatorname{Vol}\left(B_{0}\right)=\frac{1}{2} \alpha(n)\right)$, using the previous inequality and combining the constants we get $\frac{1}{2} \alpha(n) \leqslant \int_{B_{0}} f^{2}(x) d_{0} x$ with equality holding if and only if $f(x) \equiv 1$, that is $B$ is isometric to $B_{0}$.

At this point we need the assumption $n \geqslant 3$. By a Hölder inequality we have

$$
\operatorname{Vol}\left(B_{0}\right)=\frac{1}{2} \alpha(n) \leqslant \int_{B_{0}} f^{2}(x) d_{0} x \leqslant\left\{\int_{B_{0}} f^{n-1}(x) d_{0} x\right\}^{2 / n-1}\left\{\operatorname{Vol}\left(B_{0}\right)\right\}^{n-3 / n-1}
$$


thus

$$
\operatorname{Vol}\left(B_{0}\right) \leqslant \int_{B_{0}} f^{n-1}(x) d x=\operatorname{Vol}(B)
$$

with equality if and only if $B$ is isometric to $B_{0}$.

ExAMPLES. Take $Q$ large, to be chosen later depending only on $\varepsilon$. Let $f$ be a smooth function on $[-\pi / 2, \pi / 2]$ such that

(a) $f(x)=f(-x)$,

(b) $f(x)=1$ for $|x|<1 / Q^{4}$,

(c) $f(x)=Q$ for $|x| \geqslant 1 / Q^{2}$,

(d) $1 \leqslant f(x) \leqslant Q$ for all $x$.

(See figure.)

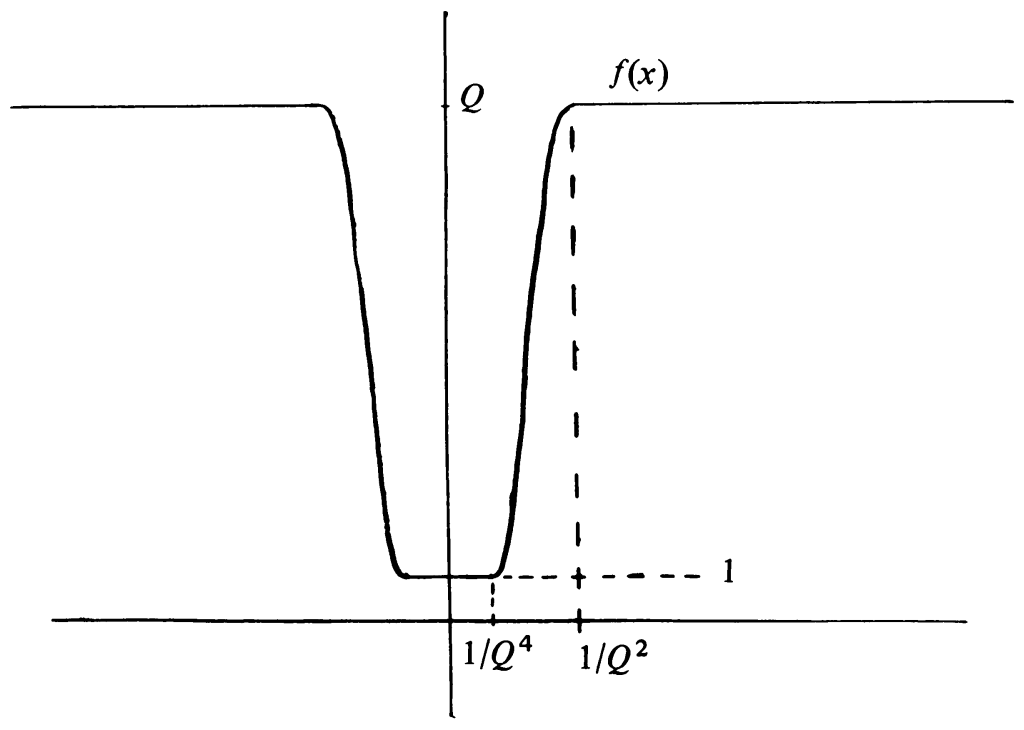

Let $g_{\varepsilon}(x)$ be the solution to

$$
g^{\prime \prime}-\left(\frac{f^{\prime \prime}}{f}\right) g=0, \quad g(0)=0, \quad g^{\prime}(0)=1,
$$

that is

$$
g_{\varepsilon}(x)=f(x) \int_{0}^{x} \frac{d t}{f^{2}(t)} .
$$

The metric $d s^{2}=d r^{2}+g_{\varepsilon}^{2}(r) d \theta^{2}$ is clearly smooth away from the origin, but the fact that $f(x)=1$ for $x$ near 0 shows that the metric is the flat metric near the origin.

We now show that since $f$ never vanishes there are no conjugate points along a diameter. Since the metric is rotationally symmetric every Jacobi field along a diameter that vanishes somewhere is of the form $J(r) X(r)$ where $J(r)$ is a function 
and $X(r)$ is a parallel unit vector field. For those Jacobi fields perpendicular to diameters $J(r)$ must satisfy

$$
0=J^{\prime \prime}-\frac{g_{\varepsilon}^{\prime \prime}}{g_{\varepsilon}} J=J^{\prime \prime}-\frac{f^{\prime \prime}}{f} J
$$

Since $f$ never vanishes $J$ cannot vanish twice (between any two zeros of $J$ there must be a zero of $f$ ). Thus there are no conjugate points along diameters.

To estimate the volume $U$ we first estimate $g(x)$.

For $x \geqslant 1 / Q^{2}$,

$$
g(x)=Q\left[\int_{0}^{1 / Q^{2}} \frac{d t}{f^{2}(t)}+\int_{1 / Q^{2}}^{x} \frac{d t}{Q^{2}}\right]<Q\left[1 / Q^{2}+x / Q^{2}\right]=\frac{x+1}{Q} .
$$

For $x \leqslant 1 / Q^{2}$,

$$
g(x)=f(x) \int_{0}^{x} \frac{d t}{f^{2}(t)}<Q X .
$$

Thus for the volume we get

$$
\begin{aligned}
U & =2 \pi \int_{0}^{\pi / 2} g^{n-1}(x) d x \\
& \leqslant 2 \pi\left[Q^{n-1} \int_{0}^{1 / Q^{2}} x^{n-1} d x+\frac{1}{Q^{n-1}} \int_{1 / Q^{2}}^{\pi / 2}(x+1)^{n-1} d x\right] \\
& <2 \pi\left[Q^{n-1} / n Q^{2 n}+\frac{1}{n Q^{n-1}}\left[\left(\frac{\pi}{2}+1\right)^{n}-\left(1+1 / Q^{2}\right)^{n}\right]\right],
\end{aligned}
$$

which can be made less than $\varepsilon$ by choice of $Q$ large.

\section{REFERENCES}

[B1] M. Berger, Some relations between volume, injectivity radius, and convexity radius in riemannian manifolds, Differential Geometry and Relativity (Cahen, Flato, eds.), Reidel, Dordrecht and Boston, 1976.

[B2] Volume et rayon d'injectivité dans les variétés riemanniennes de dimension 3, Osaka. J. Math. 14 (1977), 191-200.

[B3] Une borne inférieure pour le volume d'une variété riemannienne en function du rayon d'injectivité, Ann. Inst. Fourier (Grenoble) 30 (1980), 259-265.

[B4] _. Aire des disques et rayon d'injectivité dans les variétés riemanniennes, C. R. Acad. Sci. Paris Sér. I 292 (1981), 291-293.

[Be] A. Besse, Manifolds all of whose geodesics are closed, Ergeb. Math. Grenzgeb., vol. 93, SpringerVerlag, Berlin, 1978.

[BK] M. Berger and J. L. Kazdan, A Sturm-Liouville inequality with applications to an isoperimetric inequality for volume in terms of injectivity radius, and to Wiedersehen manifolds, General Inequalities 2 (Proc. Second Internat. Conf. General Inequalities, 1978) (E. F. Beckenbach, ed.), ISNM47, BirkhauserVerlag, Basel, 1980, pp. 367-377.

[C] C. Croke, Some isoperimetric inequalities and eigenvalue estimates, Ann. Sci. École Norm. Sup. (4) 13 (1980), 419-435.

Department of Mathematics, University of Pennsylvania, Philadelphia, Pennsylvania 19104 